\title{
Decoupling porosity and compositional effects on desilicated ZSM-5 zeolites for optimal alkylation performance
}

\section{Journal Article}

Author(s):

Milina, Maria; Mitchell, Sharon; Trinidad, Zair D.; Verboekend, Danny; Pérez-Ramírez, Javier

Publication date:

2012-04-01

Permanent link:

https://doi.org/10.3929/ethz-a-010782786

Rights / license:

In Copyright - Non-Commercial Use Permitted

Originally published in:

Catalysis Science \& Technology 2(4), https://doi.org/10.1039/c2cy00456a

Funding acknowledgement:

134572 - A fundamental approach to the scale up of hierarchical zeolite catalysts (SNF) 


\title{
Decoupling porosity and compositional effects in desilicated ZSM-5 for optimal alkylation performance
}

\author{
Maria Milina, Sharon Mitchell, Zair Domínguez Trinidad, Danny Verboekend and Javier Pérez- \\ Ramírez*
}

\author{
${ }_{5}$ Received (in $\left.X X X, X X X\right)$ Xth $X X X X X X X X X 20 X X$, Accepted Xth $X X X X X X X X X 20 X X$ \\ DOI: $10.1039 / b 000000 x$
}

\begin{abstract}
Desilication of conventional zeolites in alkaline medium generates intracrystalline mesoporosity, but inevitably changes other properties such as the Si/Al ratio and aluminium distribution. Assessing the individual effects of porosity, composition, and acidity on the catalytic performance of desilicated zeolites

10 is relevant for their optimal design. Herein, we decouple the respective impacts in the acid-catalysed alkylation of toluene (or cyclohexylbenzene) with benzyl alcohol. These reactions experience strong accessibility constraints to the micropores, providing high sensitivity to the properties of the developed mesopore surface. Through strategic comparison of alkaline-treated ZSM-5 zeolites prepared with and without subsequent acid treatment, we show that while acidity is important, the alkylation activity is

15 dominated by the mesoporous surface area. The selectivity to (methylbenzyl)benzene does not depend on the available external surface. Large mesopore volumes offer no catalytic benefit, and variation in micropore volume has a minimal effect. Acid-treated mesoporous zeolites exhibit higher catalytic activities primarily due to textural enhancements by removal of aluminium-rich amorphous debris. The catalytic results are rationalised on the basis of extensive characterisation (AAS, $\mathrm{N}_{2}$ sorption, XRD, TEM, ${ }_{20}^{27} \mathrm{Al}$ MAS NMR, FTIR, NH3-TPD) and adsorption of toluene and cyclohexylbenzene.
\end{abstract}

\section{Introduction}

Zeolites are a unique class of crystalline microporous materials owing to their high surface area, strong acidity, shape selectivity, and thermal stability. However, active sites confined within their 25 micropores are often inefficiently used due to diffusion constraints. ${ }^{1}$ Performance enhancements observed on application of hierarchical (mesoporous) zeolites, possessing a complementary network of mesopores, have attracted widespread attention. ${ }^{2-6}$

30 Interconnected mesopores can be efficiently introduced in a scalable manner by desilication of zeolites in alkaline media. ${ }^{78} \mathrm{~A}$ major concern of post-synthetic methods are the compositional changes experienced during demetallation. ${ }^{7}$ Upon alkaline treatment, silicon is preferentially extracted into solution, while 35 aluminium species realuminate on the external surface, playing an important role in pore direction. ${ }^{9,10}$ Alkaline-treated zeolites exhibit a decreased bulk $\mathrm{Si} / \mathrm{Al}$ ratio, micropore volume and crystallinity, and an increased amount of Lewis acid sites. ${ }^{11-16}$

To avoid possible alteration of the catalytic properties arising 40 from alkali-induced surface realumination, sequential acid treatments are increasingly applied. The additional step aims to restore the original framework composition. ${ }^{9,10}$ Acid washing of alkaline-leached ZSM-5 is accompanied by simultaneous enhancements in micropore volume, mesoporous surface area, 45 and crystallinity. ${ }^{11}$ These concomitant changes were never systematically studied in catalysed reactions. As such the implications of realuminated species are still not fully understood. This is particularly true for zeolites of low Si/Al ratio at high base concentrations, where larger amounts of aluminium50 rich amorphous debris are generated. ${ }^{11}$

Herein, the alkylations of toluene and cyclohexylbenzene with benzyl alcohol (Scheme 1) are taken as model reactions to decouple the relative impacts of compositional and porosity effects in desilicated zeolites. Mesoporous ZSM-5 zeolites have 55 been advantageously applied for both the alkylation ${ }^{17-19}$ and the related acylation ${ }^{20}$ of aromatic molecules. The choice of accesslimited reactions provides optimal sensitivity to the redistribution of aluminium on alkaline treatment and acid washing through confinement of the alkylation activity to the mesoporous surface. 60 Property-function relationships are established through detailed sample characterisation using multiple techniques. We address important design aspects of mesoporous zeolites such as the aluminium content and acid site speciation, the optimal extent and appropriate descriptors of mesoporosity, and the impact of 65 residual aluminium-rich species on adsorption and catalysis.

\section{Experimental}

\subsection{Post-synthetic treatments}

Various commercial ZSM-5 zeolites were obtained in the ammonium form: Z10 (PZ2/23, Zeochem), Z15 (CBV 3024E, ${ }_{70}$ Zeolyst International), Z25 (CBV 5524G, Zeolyst International), 
(a)



(b)



Scheme 1 Alkylation of (a) toluene and (b) cyclohexylbenzene with benzyl alcohol.

5 Z40 (CBV 8014, Zeolyst International), and Z200 (PZ2/400, Zeochem). The $x$ in the code $\mathrm{Z} x$ refers to the nominal Si/Al ratio according to manufacturers' specifications. The zeolites were converted into the protonic form by calcination in static air at $823 \mathrm{~K}$ for $5 \mathrm{~h}$ using a heating rate of $5 \mathrm{~K} \mathrm{~min}^{-1}$. The parent 10 samples (coded $\mathrm{P}$ ) were treated in aqueous $\mathrm{NaOH}$ solution (0.1-0.7 M, $30 \mathrm{~cm}^{3}$ per gram of zeolite) using an Easymax ${ }^{\mathrm{TM}} 102$ reactor system (Mettler Toledo). Following zeolite addition, the alkaline solution, heated at $338 \mathrm{~K}$, was stirred for $30 \mathrm{~min}$. The treatment was quenched using an ice-water mixture, and the 15 solids were collected by vacuum filtration, washed extensively with distilled water, and dried at $338 \mathrm{~K}$. Selected alkaline-treated samples were treated in aqueous $\mathrm{HCl}$ solution (0.02-0.1 M, $100 \mathrm{~cm}^{3}$ per gram of zeolite) at $338 \mathrm{~K}$ for $6 \mathrm{~h}$. The alkaline- and acid-treated zeolites were converted into the protonic form by 20 three consecutive ion exchanges in aqueous $\mathrm{NH}_{4} \mathrm{NO}_{3}$ solution $\left(0.1 \mathrm{M}, 298 \mathrm{~K}, 12 \mathrm{~h}, 100 \mathrm{~cm}^{3}\right.$ per gram of zeolite), followed by the above-described calcination step. Steaming was carried out in a quartz fixed-bed reactor (9 $\mathrm{mm}$ i.d.) with a shallow bed of zeolite powder at ambient pressure. The zeolite was treated in a 25 flow of water vapour and $\mathrm{N}_{2}$ at $873 \mathrm{~K}$ for $5 \mathrm{~h}\left(30 \mathrm{~cm}^{3} \mathrm{~min}^{-1} \mathrm{~N}_{2}\right.$, $\mathrm{H}_{2} \mathrm{O}$ partial pressure of 300 mbar). Along the manuscript, the codes AT, AW, and ST denote alkaline treatment, acid washing, and steaming, respectively.

\subsection{Characterisation methods}

30 Chemical composition of the samples was determined by atomic absorption spectroscopy (AAS) using a Varian SpectrAA 220 FS spectrometer. $\mathrm{N}_{2}$ sorption isotherms at $77 \mathrm{~K}$ were measured in a Quantachrome Quadrasorb-SI gas adsorption analyser. Prior to the measurement, the samples were degassed in vacuum at $573 \mathrm{~K}$ 35 for 10 h. X-ray diffraction (XRD) was undertaken using a PANalytical X'Pert PRO-MPD diffractometer operated in BraggBrentano geometry using Ni-filtered $\mathrm{Cu} \mathrm{K} \alpha$ radiation $(\lambda=0.1541 \mathrm{~nm})$. Data were recorded in the range $5-50^{\circ} 2 \theta$ with an angular step size of $0.05^{\circ}$ and a counting time of $8 \mathrm{~s}$ per step. ${ }_{40}{ }^{27} \mathrm{Al}$ magic angle spinning nuclear magnetic resonance (MAS NMR) spectra were recorded at a spinning speed of $12 \mathrm{kHz}$ on a Bruker AVANCE 400 NMR spectrometer equipped with a $4 \mathrm{~mm}$ probe head and $4 \mathrm{~mm} \mathrm{ZrO}_{2}$ rotors at $104.3 \mathrm{MHz} .{ }^{27} \mathrm{Al}$ spectra were measured using 4096 accumulations, $1 \mu$ s pulses, a recycle 45 delay of $0.5 \mathrm{~s}$, and $\left(\mathrm{NH}_{4}\right) \mathrm{Al}\left(\mathrm{SO}_{4}\right)_{2} \cdot 12 \mathrm{H}_{2} \mathrm{O}$ as the reference. Transmission electron microscopy (TEM) was performed with a Phillips CM12 microscope operated at $100 \mathrm{kV}$. Infrared spectroscopy was performed at $473 \mathrm{~K}$ under a $\mathrm{N}_{2}$ atmosphere using a Thermo Nicolet 5700 spectrometer equipped with a
${ }_{50}$ SpectraTech Collector II diffuse reflectance accessory and a hightemperature cell. Prior to the measurement, the sample was dried at $573 \mathrm{~K}$ under a $\mathrm{N}_{2}$ flow $\left(100 \mathrm{~cm}^{3} \mathrm{~min}^{-1}\right)$ for $60 \mathrm{~min}$. Spectra were recorded in the range of $650-4000 \mathrm{~cm}^{-1}$ with a nominal resolution of $4 \mathrm{~cm}^{-1}$ and a co-addition of 200 scans. Temperature55 programmed desorption of ammonia ( $\left.\mathrm{NH}_{3}-\mathrm{TPD}\right)$ was carried out in a Thermo TPDRO 1100 unit equipped with a thermal conductivity detector. The zeolite powder $(0.1 \mathrm{~g})$ was pretreated at $823 \mathrm{~K}$ in $\mathrm{He}$ flow $\left(20 \mathrm{~cm}^{3} \mathrm{~min}^{-1}\right)$ for $2 \mathrm{~h}$. Afterwards, 10 vol. $\% \mathrm{NH}_{3}$ in He $\left(20 \mathrm{~cm}^{3} \mathrm{~min}^{-1}\right)$ was adsorbed at $473 \mathrm{~K}$ for $6030 \mathrm{~min}$ followed by He purging at the same temperature for $1 \mathrm{~h}$. This procedure was repeated three times. Desorption of $\mathrm{NH}_{3}$ was monitored in the range $473-973 \mathrm{~K}$ using a heating rate of $10 \mathrm{~K} \mathrm{~min}^{-1}$. The adsorption isotherms of toluene and cyclohexylbenzene were measured in an Intelligent Gravimetric 65 Analyser (IGA-002, Hiden Analytical) at 298 and $320 \mathrm{~K}$, respectively. Prior to analysis, the samples $(10 \mathrm{mg})$ were outgassed at $573 \mathrm{~K}$ for $10 \mathrm{~h}$. The upper pressure limit was determined by the saturated vapour pressure of toluene and cyclohexylbenzene (38 mbar and 0.37 mbar, respectively), which 70 was estimated using the Antoine equation with the componentspecific constants reported elsewhere. ${ }^{21,22}$

\subsection{Catalytic tests}

The alkylation of toluene or cyclohexylbenzene with benzyl alcohol was conducted in an Endeavor ${ }^{\circledR}$ Catalyst Screening 75 System (Argonaut Technologies), consisting of 8 parallel reactors with a working volume of $5 \mathrm{~cm}^{3}$. The powdered catalyst $(25 \mathrm{mg}$, pre-dried at $573 \mathrm{~K}$ for $2 \mathrm{~h}$ ) was added to a mixture containing toluene (47 mmol, Fluka, 99.7\%) or cyclohexylbenzene (0.4 mmol, ABCR, 98\%), benzyl alcohol (0.6 mmol, Aldrich, 80 99.9\%), and ethylycyclohexane (0.2 mmol, Acros, > 99\%) which acted as an internal standard. The reactor was then sealed, pressurised to $5 \mathrm{bar}$, and heated to $433 \mathrm{~K}$. On reaching the desired temperature, the reaction mixture was mechanically stirred (1000 rpm) by overhead impellers. Following the desired reaction 85 time, the reactors were cooled and liquid samples were analysed using a gas chromatograph (HP 6890) equipped with a HP5 capillary column and a flame ionization detector (FID).

\section{Results and discussion}

\subsection{Alkylation over conventional zeolites}

90 Table 1 summarises the chemical composition and textural properties of the commercial H-ZSM-5 zeolites. Sharp reflections corresponding to the MFI structure were measured by XRD (not shown). As illustrated for Z15-P and Z40-P in Fig. 1, the type I $\mathrm{N}_{2}$ sorption isotherms confirmed their microporous character. The 95 corresponding micropore volumes ( $\left.V_{\text {micro}}\right)$ and low-to-moderate external surface areas ( $S_{\text {meso }}$ ) estimated are typical of ZSM-5. No evidence of intracrystalline mesoporosity was visible by TEM (not shown), or by analysis of the BJH pore size distributions (inset, Fig. 1). ${ }^{27} \mathrm{Al}$ MAS NMR spectra showed that the zeolites 100 contained predominantly tetrahedral aluminium (band at 59 ppm in Fig. 2). Only a small but identifiable proportion of octahedral aluminium, in extra-framework positions (band centred at0 ppm), was observed for zeolites with low Si/Al ratios. The increased intensities of the high-temperature desorption peak (> $700 \mathrm{~K})$, in 105 the $\mathrm{NH}_{3}$-TPD profile (Fig. 3), and of the absorbance 
Table 1 Treatment conditions and physico-chemical characterisation of parent and modified ZSM-5 zeolites.

\begin{tabular}{|c|c|c|c|c|c|c|c|c|}
\hline Sample & $\begin{array}{c}\mathrm{NaOH} \\
(\mathrm{M})\end{array}$ & $\begin{array}{l}\mathrm{HCl} \\
(\mathrm{M})\end{array}$ & $\begin{array}{l}\text { Yield } \\
(\%)\end{array}$ & $\begin{array}{c}\mathrm{Si} / \mathrm{Al}^{a} \\
(-)\end{array}$ & $\begin{array}{c}S_{\text {meso }}{ }^{b} \\
\left(\mathrm{~m}^{2} \mathrm{~g}^{-1}\right)\end{array}$ & $\begin{array}{c}S_{\mathrm{BET}}{ }^{c} \\
\left(\mathrm{~m}^{2} \mathrm{~g}^{-1}\right)\end{array}$ & $\begin{array}{c}V_{\text {micro }} b \\
\left(\mathrm{~cm}^{3} \mathrm{~g}^{-1}\right)\end{array}$ & $\begin{array}{c}V_{\text {meso }}{ }^{d} \\
\left(\mathrm{~cm}^{3} \mathrm{~g}^{-1}\right)\end{array}$ \\
\hline$\overline{Z 10-P}$ & - & - & - & - & 102 & 394 & 0.12 & 0.34 \\
\hline Z15-P & - & - & - & 15 & 76 & 412 & 0.14 & 0.15 \\
\hline Z15-ST & - & - & 100 & 15 & 112 & 415 & 0.13 & 0.23 \\
\hline Z15-AT1 & 0.6 & - & 70 & 10 & 147 & 444 & 0.13 & 0.38 \\
\hline Z15-AT1-AW1 & 0.6 & 0.02 & $94(66)^{e}$ & 12 & 158 & 588 & 0.15 & 0.40 \\
\hline Z25-P & - & - & - & 25 & 76 & 461 & 0.17 & 0.31 \\
\hline Z40-P & - & - & - & 39 & 78 & 468 & 0.17 & 0.28 \\
\hline Z40-ST & - & - & 100 & 39 & 72 & 456 & 0.17 & 0.13 \\
\hline Z40-AT1 & 0.1 & - & 84 & 34 & 135 & 497 & 0.15 & 0.19 \\
\hline Z40-AT1-AW2 & 0.1 & 0.1 & $95(80)$ & 34 & 135 & 498 & 0.15 & 0.21 \\
\hline Z40-AT2 & 0.2 & - & 67 & 25 & 288 & 589 & 0.13 & 0.40 \\
\hline Z40-AT2-AW2 & 0.2 & 0.1 & $91(61)$ & 32 & 296 & 573 & 0.12 & 0.46 \\
\hline Z40-AT3 & 0.3 & - & 45 & 17 & 417 & 637 & 0.09 & 0.78 \\
\hline Z40-AT3-AW2 & 0.3 & 0.1 & $89(40)$ & 32 & 440 & 675 & 0.10 & 0.84 \\
\hline Z40-AT3-AW2-ST & 0.3 & 0.1 & $100(40)$ & 32 & 484 & 648 & 0.06 & 0.96 \\
\hline Z40-AT4 & 0.5 & - & 18 & 7 & 336 & 519 & 0.08 & 0.98 \\
\hline Z40-AT4-AW2 & 0.5 & 0.1 & $85(15)$ & - & 415 & 635 & 0.09 & 1.00 \\
\hline Z40-AT5 & 0.7 & - & 11 & 4 & 294 & 349 & 0.02 & 0.94 \\
\hline Z40-AT5-AW2 & 0.7 & 0.1 & $69(8)$ & - & 381 & 639 & 0.11 & 1.02 \\
\hline Z200-P & - & - & - & - & 73 & 398 & 0.15 & 0.24 \\
\hline
\end{tabular}

${ }^{a}$ AAS. ${ }^{b} t$-plot method. ${ }^{c}$ BET method. ${ }^{d} V_{\text {meso }}=V_{\text {pore }}-V_{\text {micro }}{ }^{e}$ values in parentheses represent the overall yield (alkaline treatment and acid washing). The code of the samples includes the nominal Si/Al molar ratio of starting zeolites and the treatment conducted: AT (alkaline treatment), AW (acid washing), 5 and ST (steaming). P refers to the parent sample.



Fig. $1 \mathrm{~N}_{2}$ isotherms of selected zeolites. Insets: BJH mesopore size 10 distributions.

at $3600 \mathrm{~cm}^{-1}$, in the hydroxyl stretching region of the IR spectra (Fig. 4), are consistent with an increased concentration of strong (Brønsted) acid sites at lower $\mathrm{Si} / \mathrm{Al}$ ratios. The catalytic dependence on framework composition and related acidity was 15 verified by assessing the performance of the parent zeolites in the alkylation of toluene with benzyl alcohol. The conversion of benzyl alcohol $\left(X_{\mathrm{BA}}\right)$ increased linearly with the aluminium content (Fig. 5). High-silica Z200-P was catalytically inactive (a)



Fig. $2{ }^{27} \mathrm{Al}$ MAS NMR spectra of selected zeolites.

under the conditions studied.

The steam-induced extraction of framework aluminium permits determination of the influence of acidity modification on 25 the alkylation activity. Following steam treatment, no major variation in sample porosity was observed (Table 1). The ${ }^{27} \mathrm{Al}$ MAS NMR spectra, however, evidenced an increased intensity of the band at $0 \mathrm{ppm}$, and the appearance of an additional broad signal visible as a shoulder to the band at 30 $59 \mathrm{ppm}$ in the steamed zeolites (Fig. 2). These observations are related to dealumination of the framework, the condensation of dislodged aluminium as extra-framework species, and to greater variation in the coordination environment of the remaining lattice 
(a)



Fig. $3 \mathrm{NH}_{3}$-TPD profiles of selected zeolites.

(a)



Fig. 4 FTIR spectra of selected zeolites.

aluminium. $^{23,24}$ Accordingly, a decrease in the infrared absorbance at $3600 \mathrm{~cm}^{-1}$ (associated with Brønsted acid sites) ${ }^{25}$ and an increase in the absorbances at $3650 \mathrm{~cm}^{-1}$ and at $3780 \mathrm{~cm}^{-1}$ (associated with extra-framework aluminium species) ${ }^{25}$ were seen 10 in the FTIR spectrum of Z15-ST and Z40-ST (Fig. 4). Furthermore, the contribution above $700 \mathrm{~K}$ in the $\mathrm{NH}_{3}$-TPD profiles vanished (Fig. 3). No benzyl alcohol conversion was observed over the steamed zeolites (Fig. 5), strongly suggesting that weakly acidic extra-framework aluminium is not catalytically 15 active.

\subsection{Post-synthetic modification by alkaline and acid treatments}

In view of the acidity requirement identified in Section 3.1, the Z15-P and Z40-P samples were selected for the preparation of 20 hierarchical zeolites by post-synthetic modification. We have previously mapped out the range of achievable porous properties in MFI zeolites on variation of the base concentration during alkaline treatment and when treating parent zeolites with differing $\mathrm{Si} / \mathrm{Al}$ ratios. ${ }^{11}$ For aluminium-rich zeolites (e.g. $\mathrm{Si} / \mathrm{Al}=15$ ), the 25 great importance of subsequent acid washing to increase the micropore volume and mesoporous surface area and to restore the bulk Si/Al ratio of the parent zeolites, was also shown. Taking



30 Fig. 5 Influence of the molar $\mathrm{Si} / \mathrm{Al}$ ratio of the parent zeolites on the conversion of benzyl alcohol (BA) after $25 \mathrm{~min}$ in the alkylation with toluene $(\mathrm{T})$. The zero conversion on the steamed zeolites is indicated by the solid symbols. Conditions: $T=433 \mathrm{~K}, P=5$ bar, T/BA $=80,0.6 \mathrm{wt} . \%$ zeolite.

35 these observations into account, a number of hierarchical zeolites with distinct properties were prepared, characterised, and evaluated in the liquid-phase alkylation of aromatics. The corresponding chemical composition, porous properties, and treatment yields, are collected in Table 1.

40

\section{Alkaline treatment}

Upon alkaline treatment of Z15-P and Z40-P mesoporous surface areas were developed in the range of $76-147 \mathrm{~m}^{2} \mathrm{~g}^{-1}$ and $78-$ $417 \mathrm{~m}^{2} \mathrm{~g}^{-1}$, respectively (Table 1 ). As reported previously, ${ }^{11}$ 45 treatment of the zeolite with lower aluminium content (Z40-P) and at higher $\mathrm{NaOH}$ concentration led to decreased yields. Detailed characterisation is illustrated for Z15-AT1 and Z40-AT3, the hierarchical zeolites which exhibited the highest $S$ meso.

50 The introduction of mesoporosity was clearly reflected in the $\mathrm{N}_{2}$ adsorption isotherms, by an enhanced uptake at intermediate and high relative pressures (Fig. 1). Analysis of the BJH pore size distributions (Fig. 1, inset) evidenced mesopore sizes centred around $10 \mathrm{~nm}$ for Z40-AT3. For Z15-AT1, a broader mesopore 55 size distribution was observed. The intracrystalline origin of the mesoporosity has been confirmed by TEM (not shown). Due to the higher external area, the intensity of the silanol band at $3740 \mathrm{~cm}^{-1}$ in the IR spectra of desilicated samples increased (Fig. 4). The XRD patterns of the treated zeolites evidenced 60 retention of the MFI structure in all cases (not shown).

Modification of the porous properties was accompanied by a reduction in the bulk $\mathrm{Si} / \mathrm{Al}$ ratios (Table 1 ). No indication of an increase in the number of Brønsted acid sites is evidenced by IR spectroscopy (Fig. 4). The rise in the intensity of the 65 low-temperature contribution (ca. $570 \mathrm{~K}$ ) in the $\mathrm{NH}_{3}-\mathrm{TPD}$ profile(Fig. 3), reveals an increased presence of sites of moderate acidic strength, which are thought to be Lewis-type and concentrated at the mesopore surface. ${ }^{26,27}$ No substantial evidence of octahedrally-coordinated aluminium was observed in the ${ }_{70}^{27} \mathrm{Al}$ MAS NMR (Fig. 2) or IR (Fig. 4) spectra, indicating the 

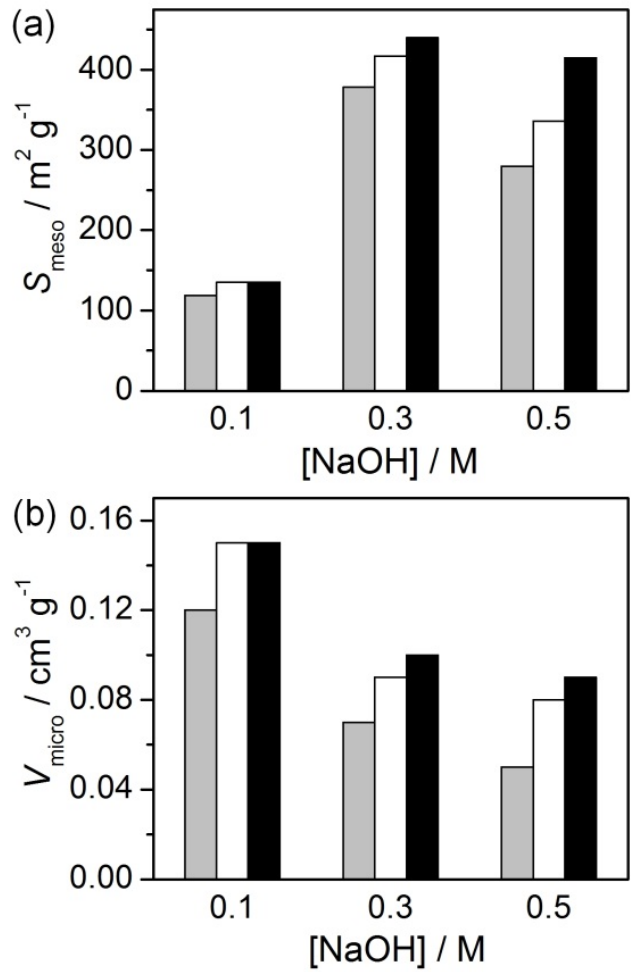

Fig. 6 Influence of ion exchange and acid washing on the porous properties of desilicated $\mathrm{Z} 40$ zeolites, treated at different $\mathrm{NaOH}$

5 concentrations. Alkaline-treated zeolites are represented by grey bars, sequentially alkaline-treated and ion-exchanged zeolites by open bars, and sequentially alkaline-treated and acid-washed zeolites by open and solid bars.

different nature in comparison with the extra-framework 10 aluminium formed upon steaming.

\section{Acid treatment}

The subsequent application of a mild acid treatment increased the molar Si/Al ratios to values similar to those of their parent 15 counterparts (Table 1). As treatment of the conventional zeolites under equivalent conditions did not extract framework aluminium, ${ }^{11,28}$ this was primarily attributed to the removal of deposited aluminium-rich debris. Consistently, the crystallinity, micropore volume, and mesoporous surface area of acid washed 20 zeolites (Z15-AT1-AW1 and Z40-AT3-AW2) increased (Table 1). Furthermore, a reduction in the low temperature desorption peak in the $\mathrm{NH}_{3}$-TPD profile (Fig. 3) and the improved visibility of the Brønsted hydroxyl stretch in the IR spectrum (Fig 4a), confirmed a decrease in the number of 25 moderately acidic sites. No significant changes were evident in the ${ }^{27} \mathrm{Al}$ MAS NMR spectra compared with the alkaline-treated sample (Fig. 2).

The mild acidic conditions ( $\mathrm{pH} \sim 5$ ) of ion-exchange (with aqueous ammonium nitrate) also contribute to variation in the 30 porous properties. The relative enhancement in $V_{\text {micro }}$ and $S_{\text {meso }}$ brought about by ion exchange and acid washing of alkalinetreated zeolites are compared in Fig. 6. For the zeolite desilicated at low base concentration $(0.1 \mathrm{M} \mathrm{NaOH})$, similar improvements (a)



(b)



35

Fig. 7 Adsorption isotherms of (a) toluene at $298 \mathrm{~K}$ and cyclohexylbenzene at $321 \mathrm{~K}$ on parent and mesoporous zeolites.

were seen for both treatments. The micropore volume was most significantly affected, increasing from 0.12 to $0.15 \mathrm{~cm}^{3} \mathrm{~g}^{-1}$, owing 40 to the exchange of charge-balancing and to the removal of residual sodium ions. Only minor variation in the mesoporous surface area was observed. In contrast for the zeolite desilicated at higher base concentration $(0.5 \mathrm{M} \mathrm{NaOH})$ acid washing was more beneficial improving $S_{\text {meso }}$ by $135 \mathrm{~m}^{2} \mathrm{~g}^{-1}$ with respect to the $4556 \mathrm{~m}^{2} \mathrm{~g}^{-1}$ increase induced by ion exchange.

\subsection{Adsorption and diffusion properties}

Further to the above described physico-chemical characterisation, the adsorption properties of Z40-P, Z40-AT3, and Z40AT3-AW2 were assessed with respect to toluene and 50 cyclohexylbenzene, the reagents subsequently used in the catalytic study.

The toluene adsorption isotherm of the parent Z40-P exhibited a single sharp uptake at low pressure ( $p<2$ mbar), related to micropore filling and characteristic of type I behaviour (Fig. 7a).

${ }_{55}$ The mesoporous zeolites displayed slightly higher initial uptakes of toluene compared to the parent sample. In contrast, analysis by $\mathrm{N}_{2}$ adsorption evidenced lower micropore volumes $\left(0.10 \mathrm{~cm}^{3} \mathrm{~g}^{-1}\right.$ compared to $0.17 \mathrm{~cm}^{3} \mathrm{~g}^{-1}$, respectively). This demonstrates an additional adsorption contribution, which should be related to the 60 mesopore introduction. At higher pressures the adsorbed amount increased progressively. The uptake of the Z40-AT3-AW2 was higher than that of Z40-AT3 at all relative pressures, consistent with the enhanced textural properties of the washed sample. Assuming a bulk liquid density of toluene, the total pore volumes ${ }_{65}$ estimated are similar to those derived from $\mathrm{N}_{2}$ adsorption. This indicates that both the micro- and mesopores were completely filled, confirming their full accessibility. 




Fig. 8 Influence of post-synthetic modification on the conversion of benzyl alcohol in the alkylation with toluene. Reaction conditions as in 5 caption of Fig. 5.

For the larger cyclohexylbenzene, which is not expected to penetrate into the micropores, significantly lower uptakes were observed for all zeolites (Fig. 7b). The mesoporous samples exhibited higher uptakes that the parent zeolite. In contrast to the 10 adsorption of toluene, the initial adsorption of cyclohexylbenzene was similar for both the alkaline-treated and acid-washed zeolites, reflecting the negligible impact of micropore volume. However, the higher mesoporosity of the washed sample resulted in increased uptake at higher pressures.

\section{3.4. Alkylation over hierarchical ZSM-5}

Fig. 8 displays the activity enhancements achieved in the alkylation of toluene with benzyl alcohol over the alkaline-treated zeolites, possessing the highest mesoporous surface areas (Z15-AT1 and Z40-AT3), with respect to their purely 20 microporous counterparts (Z15-P and Z40-P). Following $25 \mathrm{~min}$ of reaction, the corresponding benzyl alcohol conversions were 12 and 83\%, for Z15-P and Z15-AT1, and 7 and 90\%, for Z40-P and Z40-AT3, respectively. The gain in catalytic activity on application of the acid-washed hierarchical zeolites, is also 25 shown. This effect was greater for Z15-AT1 than Z40-AT3 in line with the increased presence of aluminium-rich debris evidenced in Section 3.2.

Figs. 9a-c summarise the relationship between toluene alkylation activity, the porous properties of mesoporous zeolites 30 derived from Z40-P ( $V_{\text {microo }}, S_{\text {meso }}$, and $\left.V_{\text {meso }}\right)$, and treatment efficiency as a function of the $\mathrm{NaOH}$ concentration applied in their preparation. The negligible dependence of catalytic activity on the micropore volume is consistent with the access-limited

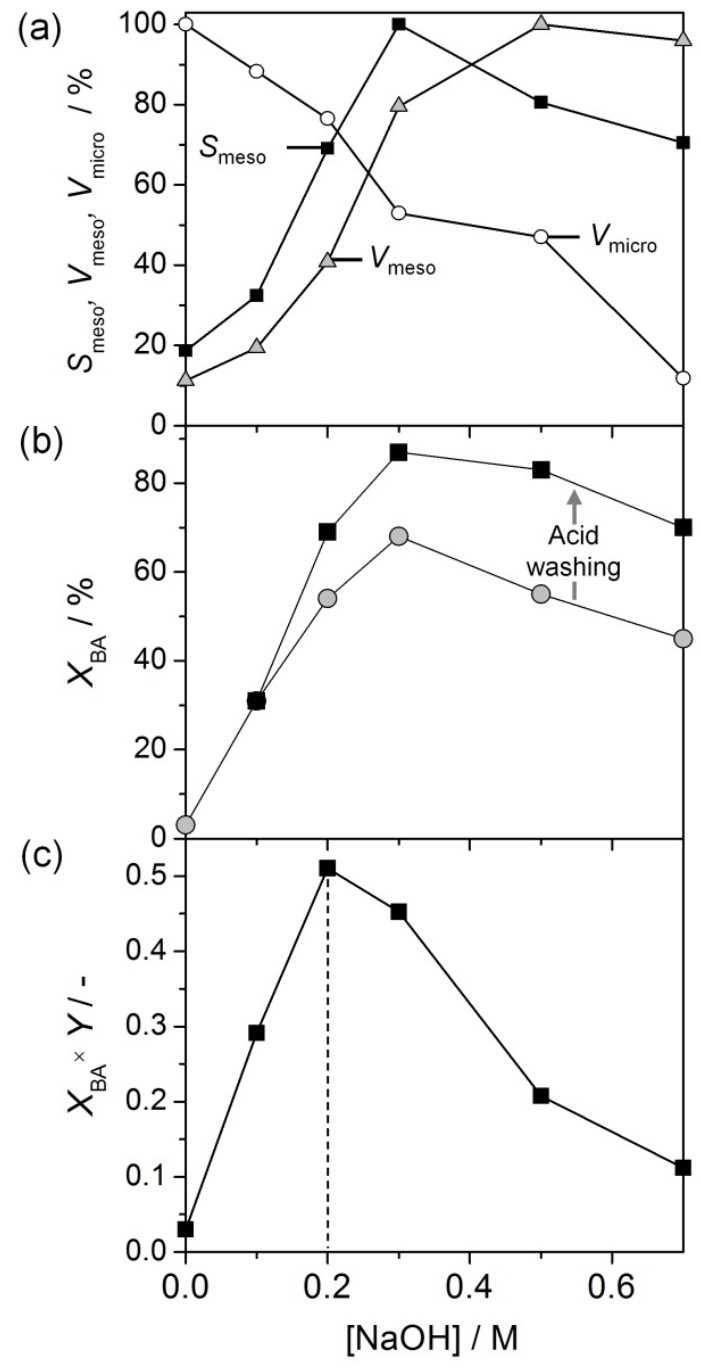

35

Fig. 9 Effect of the $\mathrm{NaOH}$ concentration on (a) the porous properties of the alkaline-treated Z40, (b) the conversion of benzyl alcohol during the alkylation with toluene over alkaline-treated (AT) zeolites before (grey circles) and after (solid squares) sequential acid washing (AW) after

4010 min of reaction, and (c) the benzyl alcohol conversion factored by the overall treatment yield for the AT-AW zeolites. Values of the parent zeolite are represented at $[\mathrm{NaOH}]=0 \mathrm{M}$. Reaction conditions as in caption of Fig. 5 .

nature of the alkylation reaction. Accordingly, the strong ${ }_{45}$ interdependence between $S_{\text {meso }}$ and $X_{\mathrm{BA}}$ is directly related to the larger surface area available for alkylation. The activity $\left(X_{\mathrm{BA}}\right.$ at $t=10 \mathrm{~min}$ ) mirrors the corresponding variation in the mesoporous surface area. Both reach a maximum for the sample treated at $0.3 \mathrm{M} \mathrm{NaOH}$. In contrast, the trend in $X_{\mathrm{BA}}$ deviates ${ }_{50}$ from that of $V_{\text {meso. }}$. This is most noticeable at increased values of mesopore volume, demonstrating that the generation of large mesopores is not advantageous in liquid-phase alkylation. Therefore, the mesopore surface seems a more suitable descriptor in the design of hierarchical zeolite catalysts than the mesopore 55 volume. The positive impact of acid washing on the catalytic activity increased with the severity of the initial alkaline treatment (Fig. 9b). This further evidences the benefits of 




Fig. 10 Conversion of benzyl alcohol in the alkylation with toluene (solid bars) and cyclohexylbenzene (open bars) over hierarchical zeolites. 5 Conditions: $T=433 \mathrm{~K}, P=5 \mathrm{bar}, t=25 \mathrm{~min}, \mathrm{~T} / \mathrm{BA}=80, \mathrm{CHB} / \mathrm{BA}=130$, 0.6 wt. $\%$ zeolite.

removing the aluminium-rich debris which block potentially active Brønsted acid sites at the pore mouths. To account for the loss of raw material which occurs during preparation, the 10 conversion of benzyl alcohol was factored by the treatment yield of hierarchical zeolites (Fig. 9c). Taking this into consideration, Z40-AT2-AW2, desilicated with $0.2 \mathrm{M} \mathrm{NaOH}$, was identified as the optimal mesoporous zeolite catalyst derived from Z40-P in this study.

15 The catalytic properties of the hierarchical zeolites were also characterised in the alkylation of cyclohexylbenzene (Fig. 10). The larger substituted aromatic molecule was expected to provide even greater sensitivity to differences in the mesoporous surface structure than toluene. Remarkably, although the conversion of

20 benzyl alcohol over Z40-AT3 was 30\% lower than observed with toluene, the catalytic activity of Z40-AT3-AW2 (after $25 \mathrm{~min}$ ) was nearly equivalent in both alkylations. This permitted further insight into the property-function relationships of the hierarchical zeolite catalysts. Acid sites present on the mesoporous surface of 25 the modified zeolites have sufficient strength to catalyse both alkylation reactions. The higher conversions with toluene over the unwashed sample implied that residual aluminium species restrict access to active sites on the mesopore surface of alkalinetreated zeolites. The removal of such species by acid washing 30 unblocks pore mouths and improves the active site accessibility, thereby reducing the molecular size-dependence of the catalytic activity. Comparatively, the relative enhancement of $X_{\mathrm{BA}}$ on mild acid treatment of Z40-AT3 was 9 and 45\% for toluene and cyclohexylbenzene, respectively.

35 Consistent with previous work, ${ }^{29}$ a clear trend is observed between alkylation activity and secondary mesoporosity (Fig. 11a). Interestingly, the linear dependence is distinct for zeolites obtained from Z15-P or Z40-P. This relates to the difference in framework aluminium content, which is 2.6 times 40 higher in Z15-P. A three times larger mesoporous surface area is required to reach equivalent benzyl alcohol conversions for the Z40-derived hierarchical zeolites. This implies that mesoporosity introduction in aluminium-rich zeolites potentially offers greater catalytic improvements. The absence of any clear correlation with ${ }_{45}$ the bulk $\mathrm{Si} / \mathrm{Al}$ ratio proves that moderately acidic species
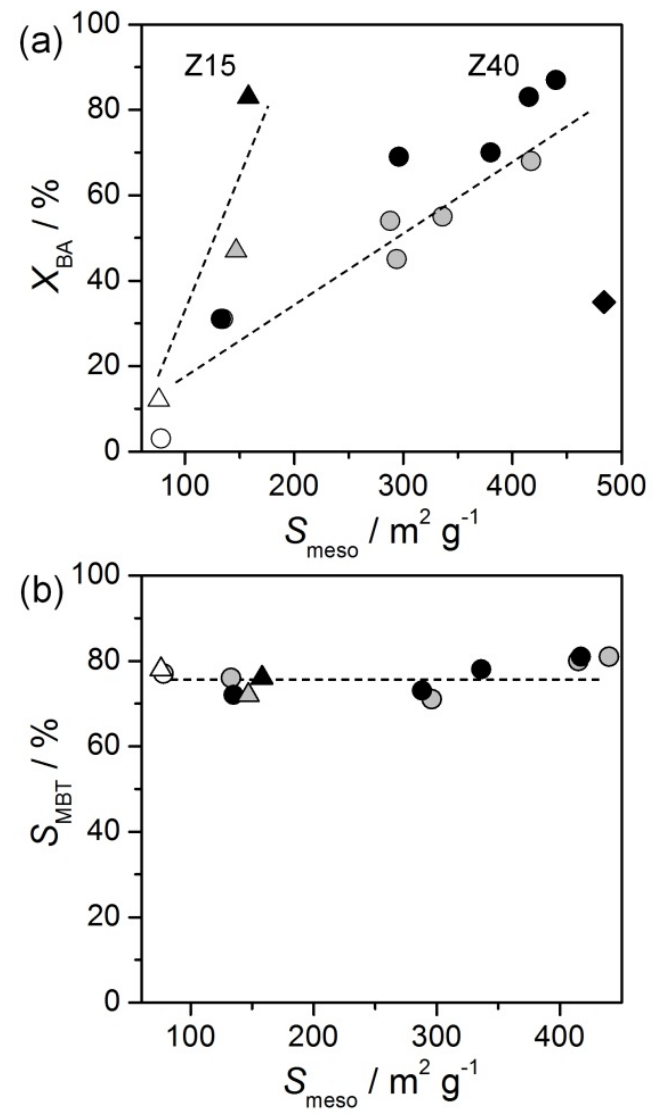

Fig. 11 (a) Conversion of benzyl alcohol and (b) selectivity to (methylbenzyl)benzene in the alkylation of toluene over zeolites derived 50 from Z15-P (triangles) and Z40-P (circles) with respect to the mesoporous surface area $\left(S_{\text {meso }}\right)$ after 10 min of reaction. Parent zeolites are indicated by open symbols, alkaline-treated by grey symbols, and acid-washed by black symbols. The black diamond symbol represents the drop in activity upon steaming of mesoporous Z40-AT3-AW2. Reaction conditions as in 55 caption of Fig. 5.

residual from alkaline treatment are less active than framework aluminium in the context of alkylation. In addition, the amount of strong acid sites in the remaining zeolite framework is apparently unaffected by the treatments applied. The selectivity for 60 (methylbenzyl)benzene was around 75\%, independent of the bulk $\mathrm{Si} / \mathrm{Al}$ ratio, the textural properties, or the presence of amorphous aluminium-rich debris (Fig. 11b). The main by-product of the reaction, dibenzyl ether (25\%), results from the self-etherification of benzyl alcohol. Polyalkylated compounds were not observed, 65 as expected from the use of an excess of toluene $(\mathrm{T} / \mathrm{BA}=80$ ). The selectivity relationship between ortho, meta, and para isomers was estimated to be 4:1:5 for all zeolites. The similar selectivities verify that no shape selectivity effects, related to the participation of micropores, influence the product distribution. In 70 agreement with our earlier observations, steaming significantly reduced the activity of the most active mesoporous zeolite (Z40-AT3-AW2 in Fig. 11a). However, the steamed sample retained a higher activity ( $X_{\mathrm{BA}}=35 \%$ after $10 \mathrm{~min}$ of reaction) than the best performing purely microporous zeolite.

75 The catalytic trends described in this work, have all been derived from liquid-phase alkylations of aromatic molecules. It 
should be pointed out that these observations can differ for other reaction systems, with distinct acidity requirements, and extent of micropore utilisation, or under different process conditions. Furthermore, more detailed evaluation of the acidic properties is 5 required to understand the variation in acid site speciation occurring upon post-synthetic modification. These aspects will be addressed in future work.

\section{Conclusions}

Both compositional and porosity variations induced by alkaline 10 treatment contribute to the catalytic performance of desilicated ZSM-5 in the liquid-phase alkylation of toluene with benzyl alcohol. Textural development, which is best described in terms of mesoporous surface area, clearly dominates the overall catalytic performance of access-limited reactions. Larger pores, 15 evidenced by high mesopore volumes and larger average pore diameters, were not beneficial in liquid-phase alkylation. In terms of compositional effects the efficiency of mesopore introduction in enhancing the catalytic activity is proportional to the framework density of aluminium in the parent zeolite. No 20 relationship is found with the bulk Si/Al ratio. Aluminium-rich species residual from desilication block active sites at the external surface, impacting the activity improvement in the alkylation. The magnitude of this detrimental effect depends on the extent of dissolution and can be compensated by subsequent acid 25 treatment. The catalytic knowledge gained in this study strengthens the ability to design hierarchical zeolites by desilication. This is achieved by defining the optimal starting zeolite and treatment conditions, and the requirement for acid washing.

\section{Acknowledgements}

ETH Zurich and the Swiss National Science Foundation (project number 200021-134572) are acknowledged for financial support.

\section{Notes and references}

Institute for Chemical and Bioengineering, Department of Chemistry and 35 Applied Biosciences, ETH Zurich, Wolfgang-Pauli-Strasse 10,

HCI E 125,CH-8093, Zurich, Switzerland. E-mail: jpr@chem.ethz.ch;

Fax:+41 446331405

1. J. Kärger and D. Freunde, Chem. Eng. Tech., 2002, 25, 769.

40 2. J. Pérez-Ramírez, C.H. Christensen, K. Egeblad, C.H. Christensen and J.C. Groen, Chem. Soc. Rev., 2008, 37, 2530.

3. R. Chal, C. Gérardin, M. Bulut and S. van Donk, ChemCatChem, 2011, 3, 67.

4. W. Schmidt, ChemCatChem, 2009, 1, 53.

45 5. M.S. Holm, E. Taarning, K. Egeblad and C.H. Christensen, Catal. Today, 2011, 168, 3.

6. J. Čejka and S. Mintova, Catal. Rev., 2007, 49, 457.

7. D. Verboekend and J. Pérez-Ramírez, Catal. Sci. Technol., 2011, 1, 879.

50 8. J. Pérez-Ramírez, S. Mitchell, D. Verboekend, M. Milina, N.-L. Michels, F. Krumeich, N. Marti and M. Erdmann, ChemCatChem, 2011, 3, 1731.

9. D. Verboekend and J. Pérez-Ramírez, Chem. Eur. J., 2011, 17, 1137.

10. C. Fernandez, I. Stan, J.-P. Gilson, K. Thomas, A. Vicente,

55 A. Bonilla and J. Pérez-Ramírez, Chem. Eur. J., 2010, 16, 6224.

11. D. Verboekend, S. Mitchell, M. Milina, J.C. Groen and J. PérezRamírez, J. Phys. Chem. C, 2011, 115, 14193.
12. D. Verboekend, A.M. Chabaneix, K. Thomas, J.-P. Gilson and J. Pérez-Ramírez, CrystEngComm., 2011, 13, 3408.

60 13. P. Sazama, B. Wichterlova, J. Dedecek, Z. Tvaruzkova, Z. Musilova, L. Palumbo, S. Sklenak and O. Gonsiorova, Microporous Mesoporous Mater., 2011, 143, 87.

14. D. Verboekend, L.A. Villaescusa, K. Thomas, I. Stan and J. PérezRamírez, Catal. Today, 2010, 152, 11.

65 15. S. Svelle, L. Sommer, K. Barbera, P.N.R. Vennestrøm, U. Olsbye, K.P. Lillerud, S. Bordiga, Y. Pan and P. Beato, Catal. Today, 2011, 168, 38.

16. D. Verboekend, R. Caicedo-Realpe, A. Bonilla, M. Santiago and J. Pérez-Ramírez, Chem. Mater., 2010, 22, 4679.

70 17. J. Pérez-Ramírez, D. Verboekend, A. Bonilla and S. Abelló, Adv. Funct. Mater., 2009, 19, 3972.

18. Z. Musilová, N. Žilkova, S.-E. Park and J. Čejka, Top Catal., 2010, 53, 1457.

19. C.H. Christensen, K. Johannsen, E. Törnqvist, I. Schmidt, H. Topsøe and C.H. Christensen, Catal. Today, 2007, 128, 117.

20. D.P. Serrano, R.A. García and D. Otero, Appl. Catal. A, 2009, 359, 69.

21. D.R. Stull, Ind. Eng. Chem., 1947, 39, 517.

22. K.S. Pitzer and D.W. Scott, J. Am. Chem. Soc., 1943, 65, 803.

80 23. J. Klinowski, Ann. Rev. Mater. Sci., 1988, 18, 189.

24. A. Samoson, E. Lippmaa, G. Engelhardt, U. Lohse and H.-G. Jerschkewitz, Chem. Phys. Lett., 1987, 134, 589.

25. J.C. Groen, J.C. Jansen, J.A. Moulijn and J. Pérez-Ramírez, J. Phys. Chem. B, 2004, 108, 13062.

85 26. M.S. Holm, S. Svelle, F. Joensen, P. Beato, C.H. Christensen, S. Bordiga and M. Bjørgen, Appl. Catal., 2009, 356, 23.

27. F. Thibault-Starzyk, I. Stan, S. Abelló, A. Bonilla, K. Thomas, C. Fernandez, J.-P. Gilson and J. Pérez-Ramírez, J. Catal., 2009, 264, 11.

90 28. R. Caicedo-Realpe and J. Pérez-Ramírez, Microporous Mesoporous Mater., 2010, 128, 91.

29. A.N.C. van Laak, S.L. Sagala, J. Zečević, H. Friedrich, P.E. de Jongh and K.P. de Jong, J. Catal., 2010, 276, 170. 